\title{
CYCLING CULTURE: FROM USERS' PERCEPTIONS TO PLANNING POLICIES
}

\author{
MARIA GRAZIA BELLIZZI, LAURA EBOLI \& CARMEN FORCINITI \\ Department of Civil Engineering, University of Calabria, Italy
}

\begin{abstract}
The current levels of air pollution and the noticeable consequences of climate change are at the heart of many social movements today. In this contest, a much faster transition towards more sustainable urban transport and land use patterns is needed. Planning policies should take more seriously actions that direct users' behavior from the use of private car to a mobility based on cycling, walking, and public transport. However, if on the one hand several case studies show how in some countries the cycling culture is really entrenched, on the other hand some contexts need more effective transport policies. In this work, the case study is represented by a little urban area of the southern Italy where about 120,000 inhabitants live. The mild climate and the flat region of this area have motivated our research. This work has the aim to understand why in an urban context, with characteristics that are suitable for the use of the bike, the cycling culture is missing. Furthermore, by analyzing users' perceptions on the existing infrastructures, we want to give a contribution to identify the right policies for promoting and managing cycling mobility. Data collected by a face-to-face survey have been used for our purposes. Four cycle paths with different characteristics are object of this study. More specifically, interviewed users were asked to provide their perceptions concerning several aspects and elements that they experienced while cycling. From the proposed analysis, it is possible to conclude that the improvement of the existent bike network could promote bike use. In addition, the results could be useful to identify convenient policies for encouraging people behavior to cycling culture.

Keywords: sustainable mobility, cycling culture, users’ perceptions, transport policies.
\end{abstract}

\section{INTRODUCTION}

Never before in recent years there has been a growing sensitivity related to the environment and its protection. Urban settlements are among the main sources of air pollution, and pollutants emitted into the atmosphere are the cause of various environmental problems as the climate change [1]. Transport sector is one of the most responsible of urban pollution. For this reason, concrete policies should be acted in transport sector, and especially in urban areas. Encouraging people to use public transport and getting around the city by active modes of transport (walking and cycling) is as useful as hard to do. Underlying this difficulty, there is what can be defined as mobility culture. Especially for cycling, it seems that in some cities there is a greater propensity to use the bike than in others. As an example, in Denmark and Sweden cycling has been a key part of urban mobility in several cities since ever [2]. On the contrary, there are other realities as the United States where cycling is an under-used mode for transportation [3]. By following the Eurobarometer report 422a [4], if Netherlands is the country with the highest rate of urban cyclists (36\%), on the other hand Italy is just below the European average $(6 \%)$ and a little better than France and Spain. However, it often happens that in these countries characterized by a car dependence, the planning policies continue to be focused on motorized transport systems [5]. These policies do not help cycling to establish itself as a valid mode of transport. In order to increase cycling in cities, investing in bicycle infrastructure is needed [6] and it is important that the expense can be justified [7]. In this context, starting from cyclists' point of view might be a smart strategy to correctly address planning policies and therefore the investments to be made. As an example, Fernández-Heredia et al. [8] conducted a survey just to understand cyclists perceptions and 
behavior with the final aim to determine the appropriate actions to encourage bicycle use. Furthermore, Damant-Sirois et al. [9] proposed different strategies to different types of cyclist by starting from their characteristics and behavior.

The present work aims to derive policies for promoting cycling by analyzing users' perceptions on existing cycle paths, placed in an urban context where the car reaches an average of $90 \%$ of motorized movements [10], although the mild climate and the flat region of the area would make these paths very suitable for cycling. Specifically, this works aims to establish the strengths and the weakness of the existing infrastructures starting from the users' point of view, with the aim to identify strategies for promoting and managing cycling mobility. On the basis of users' perceptions, it is possible to obtain targeted policies and avoid waste of financial resources. Data supporting this study were collected by face-to-face surveys conducted on four different cycle paths. The innovative aspect is represented just by the type of collected data, which relate to aspects concerning comfort, nuisance and protection on the cycle paths. Next section below provides a detailed description of the case study. Specifically, the main physical characteristics of the cycle paths are reported. Successively, the survey instrument is described, and a preliminary analysis of the sample is conducted. In the Section 3, the cycle paths are compared through the analysis of users' perceptions. Finally, in the last sections the main conclusions and final remarks are presented.

\section{CASE STUDY}

The urban area of Cosenza (Italy), originated from the union of the town of Cosenza and Rende, has been chosen as case study. The two towns are very close, so that one can be considered an extension of the other (Fig. 1).

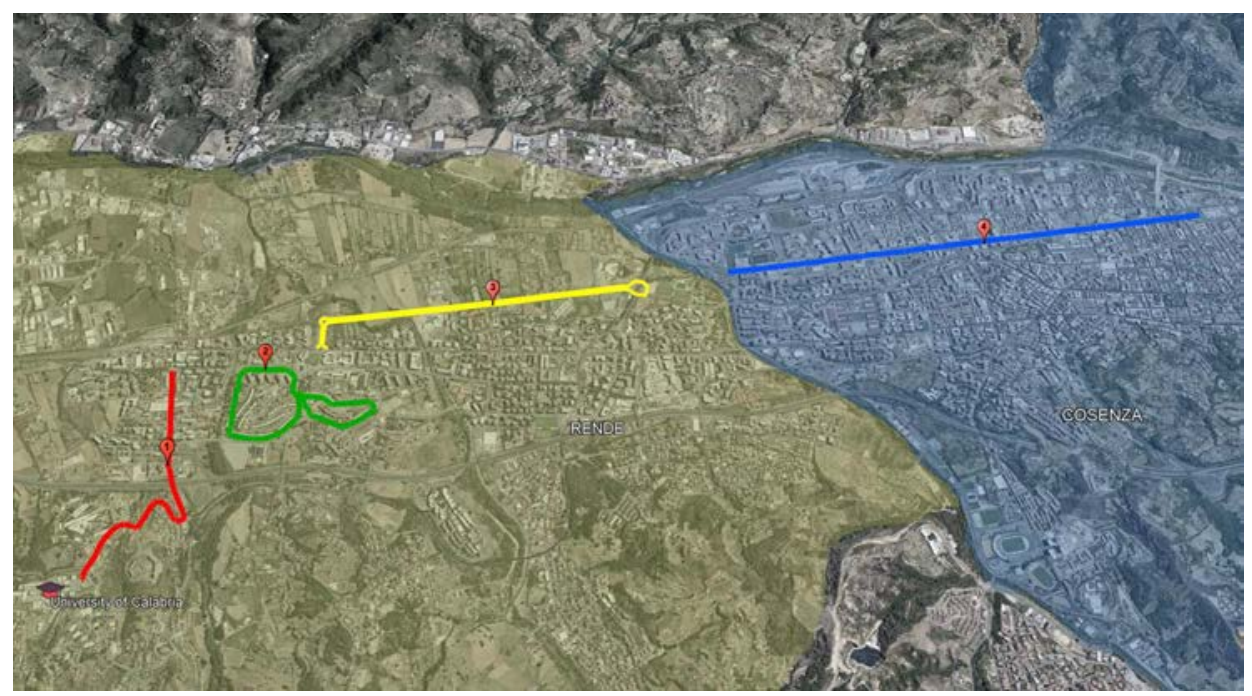

Figure 1: Cycle paths of the urban area of Cosenza.

The University of Calabria represents the main cultural center of the urban area. The University is located in Rende and attracts roughly 30,000 students and 2,000 employees, among teachers, technical and administrative staff. Thanks to the presence of the University, the urban area has a population of 120,000 inhabitants. The mild climate and the flat region 
of this urban area are supportive to non-motorized mobility. However, although there are several cycle paths in the area, the cycling culture has still not well established.

\subsection{Cycle paths}

Four cycle paths of the urban area were selected as object of this study, being the most important ones of the urban area. Specifically, three of them develop within the municipality of Rende, and one within the municipality of Cosenza (Fig. 1).

The first one (the red path) is located along an urban road that connects the residential neighborhood (named "Quattromiglia") with the university campus. This path has no real cycling infrastructure (Fig. 2(a)) and it is about $2 \mathrm{~km}$ long. However, being the main route connecting the residential area with the university campus, it is often used by students for walking or cycling. The main noticeable weaknesses of this path are: poor lighting, no separation from vehicles and not signalized road crossing. The green path develops for about $3 \mathrm{~km}$ around the social housing area of "Villaggio Europa." It is characterized by a two-way bikeway separated from the motorized-traffic, with red paving bounded by concrete curbs (Fig. 2(b)). The surrounding green areas are certainly the favorable aspect of this path.

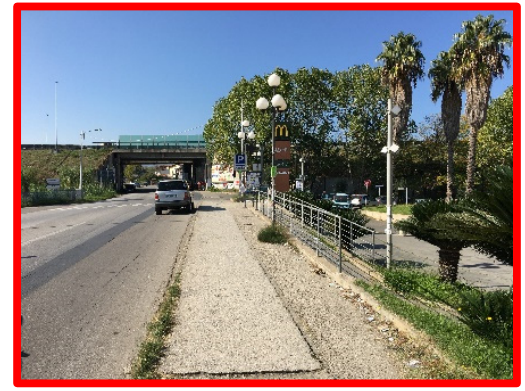

(a)

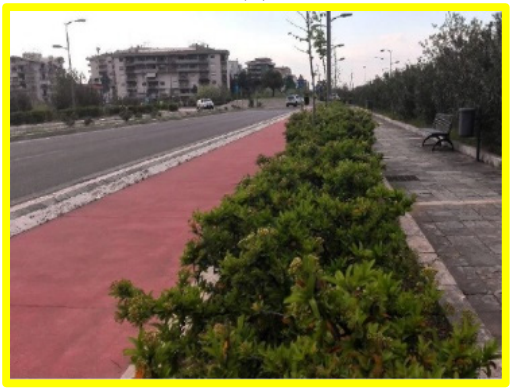

(c)

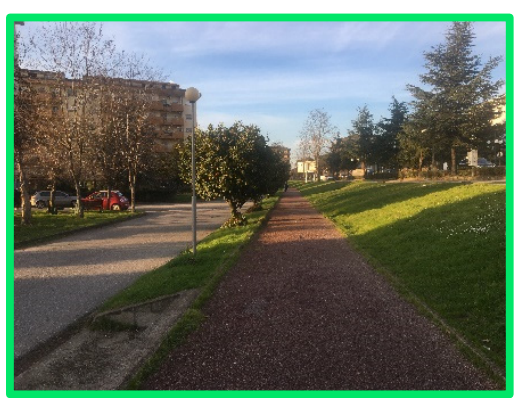

(b)

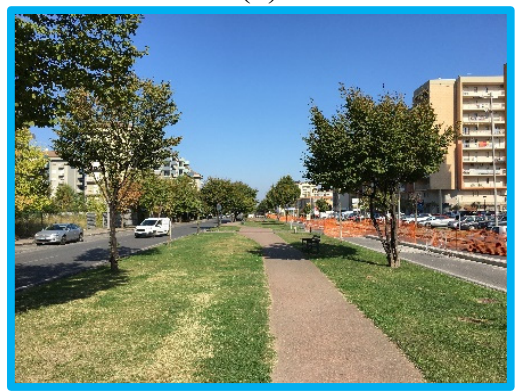

(d)

Figure 2: Present state of cycle paths. (a) Red path; (b) Green path; (c) Yellow path; and (d) Blue path.

The bikeway of the yellow path extends about $5 \mathrm{~km}$ alongside the two roadways of "Viale Principe." The bikeway has a red paving and is separated by a concrete curb from the roadway, and by a hedge from the walkway (Fig. 2(c)). The strengths of the yellow path are definitely its linearity and continuity. The blue path is roughly $3 \mathrm{~km}$ long and it is located 
alongside "Viale Mancini." Its two-way bikeway is separated from the motorized traffic by a green area with trees and benches (Fig. 2(d)).

In summary, the yellow and the blue paths are very similar to each other because of the linearity of their routes that extend along busy roads. The red and the green paths are those that differ the most. The red path runs along a busy road as well, however, it does not present a real cycling infrastructure. On the contrary, the green path is the only one located in a residential area and mainly runs along a road used by inhabitants. The four cycle paths are not connected among them despite being very close, and this aspect is certainly unfavorable for users.

\subsection{Survey}

The survey was conducted during the period from September to October 2019 by face-toface interviews. The interviews took place both in the morning (from 8:30 to 12:30) and in the afternoon (from 16:00 to 20:00). The users cycling along the paths were randomly stopped and interviewed by using a paper questionnaire. Each interview lasted about ten minutes. Once an interview was finished, the interviewer stopped the next user and started a new interview. The questionnaire derives from an ongoing research projects of the Universidad de los Andes (Bogotà, Colombia). It is structured with the aim of collecting cyclists' perceptions about the whole path and a specific point of the path. At the beginning, the interviewee was requested to express the overall level of satisfaction with the cycle path by using a 11-point Likert scale ranging from 0 (totally unsatisfied) to 10 (totally satisfied). Then, the perceptions about the whole path were requested in terms of comfort and nuisance. Specifically, the users had to express a level of comfort related to the following elements: other bikes, toilets, shops, and shady areas. The evaluation scale is a 11-point Likert scale ranging from 0 (totally uncomfortable) to 10 (totally comfortable). Regarding the degree of nuisance, the elements taken into account are: pedestrian flow, opposing pedestrians, objects obstructing the bikes passage, and parked scooters. Also in this case, the scale is a 11-point Likert scale ranging from "absence of nuisance" to "high degree of nuisance." The part concerning the users' perceptions in a specific point of the path are divided in: level of comfort, degree of nuisance and degree of protection. The evaluation scale for measuring the level of comfort and degree of nuisance are the same of those adopted for the whole path. The 11-point Likert scale related to the degree of protections ranges from 0 (absence of protection) to 10 (high degree of protection). The degree of nuisance in a specific point of the path was requested in relation to the following elements and characteristics: street lane width, traffic volume, trucks and bused volume, traffic speed, pollution, and noise. Otherwise, the level of comfort in a specific point was investigated in terms of: temperature, lighting, odor, environment closed to the cycle path, cleanliness, and landscape. Finally, the degree of protection was assessed with regard to: theft or robbery, accidents on the cycle path, accidents involving vehicles, weather, and stray animals. At the end of the questionnaire, some information about the trip (origin and destination, purpose, estimated time for reaching the destination, frequency of trip) and about the interviewee (e.g. gender, age, employment and marital status) were requested.

\subsection{Sample}

A total of 145 users were interviewed along the cycle paths. Specifically, 16 along the red path, 44 along the green path, 56 along the yellow path, and 29 along the blue one. Not all the users were willing to stop while cycling and answer the questionnaire. The highest 
response rates were recorded for the red and the blue paths ( $76.2 \%$ and $82.9 \%$ respectively). Otherwise, the response rates related to the green and yellow path are equal to $45.4 \%$ and $50.0 \%$ respectively. The willingness to respond of users is most likely due to the conditions of the cycle paths at the time of the survey. In fact, both the users of the red and blue paths have been more willing to take part at the survey because they could not cycle quickly because of the lack of a bikeway and the presence of construction going on, respectively.

In Table 1 the socio-demographic characteristics and some trip information related to the sample of each path are reported.

Table 1: Socio-demographic and trip characteristics of the sample of each cycle path.

\begin{tabular}{|c|c|c|c|c|c|c|c|c|c|}
\hline \multirow{2}{*}{ Category } & \multirow{2}{*}{ Sub-category } & \multicolumn{2}{|c|}{ Red } & \multicolumn{2}{|c|}{ Green } & \multicolumn{2}{|c|}{ Yellow } & \multicolumn{2}{|c|}{ Blue } \\
\hline & & $\mathrm{n}$ & $\%$ & $\mathrm{n}$ & $\%$ & $\mathrm{n}$ & $\%$ & $\mathrm{n}$ & $\%$ \\
\hline \multirow{2}{*}{ Gender } & Male & 15 & 93.8 & 26 & 59.1 & 31 & 55.4 & 15 & 51.7 \\
\hline & Female & 1 & 6.2 & 18 & 40.9 & 25 & 44.6 & 14 & 48.3 \\
\hline \multirow{7}{*}{ Age } & $\leq 20$ & 1 & 6.2 & 1 & 2.3 & 0 & 0.0 & 2 & 6.9 \\
\hline & $21-25$ & 12 & 75.0 & 4 & 9.1 & 7 & 12.5 & 1 & 3.4 \\
\hline & $26-30$ & 3 & 18.8 & 11 & 25.0 & 6 & 10.7 & 1 & 3.4 \\
\hline & $31-40$ & 0 & 0.0 & 8 & 18.2 & 17 & 30.4 & 13 & 44.8 \\
\hline & $41-50$ & 0 & 0.0 & 14 & 31.8 & 13 & 23.2 & 12 & 41.4 \\
\hline & $51-60$ & 0 & 0.0 & 6 & 13.6 & 11 & 19.6 & 0 & 0.0 \\
\hline & $>60$ & 0 & 0.0 & 0 & 0.0 & 2 & 3.6 & 0 & 0.0 \\
\hline \multirow{6}{*}{ Employment } & Student & 16 & 100.0 & 12 & 27.3 & 14 & 25.0 & 4 & 13.8 \\
\hline & Employee & 0 & 0.0 & 21 & 47.7 & 19 & 33.9 & 13 & 44.8 \\
\hline & Freelancer & 0 & 0.0 & 9 & 20.5 & 11 & 19.6 & 6 & 20.7 \\
\hline & Pensioner & 0 & 0.0 & 0 & 0.0 & 2 & 3.6 & 0 & 0.0 \\
\hline & Unemployed & 0 & 0.0 & 2 & 4.5 & 6 & 10.7 & 6 & 20.7 \\
\hline & Other & 0 & 0.0 & 0 & 0.0 & 4 & 7.1 & 0 & 0.0 \\
\hline \multirow{6}{*}{ Marital status } & Single & 12 & 75.0 & 14 & 31.8 & 25 & 44.6 & 5 & 17.2 \\
\hline & Cohabitation & 0 & 0.0 & 3 & 6.8 & 0 & 0.0 & 0 & 0.0 \\
\hline & Married & 0 & 0.0 & 13 & 29.5 & 17 & 30.4 & 16 & 55.2 \\
\hline & Divorced & 0 & 0.0 & 6 & 13.6 & 6 & 10.7 & 1 & 3.4 \\
\hline & Widower & 0 & 0.0 & 0 & 0.0 & 2 & 3.6 & 0 & 0.0 \\
\hline & Other & 4 & 25.0 & 8 & 18.2 & 6 & 10.7 & 7 & 24.1 \\
\hline \multirow{6}{*}{ Frequency of trip } & Once a day & 10 & 62.5 & 3 & 6.8 & 4 & 7.1 & 5 & 17.2 \\
\hline & Several times a day & 5 & 31.3 & 6 & 13.6 & 1 & 1.8 & 1 & 3.4 \\
\hline & Once a week & 1 & 6.2 & 14 & 31.8 & 32 & 57.1 & 13 & 44.8 \\
\hline & Once every 10 days & 0 & 0.0 & 9 & 20.5 & 9 & 16.1 & 2 & 6.9 \\
\hline & Rarely & 0 & 0.0 & 12 & 27.3 & 10 & 17.9 & 8 & 27.6 \\
\hline & Never & 0 & 0.0 & 0 & 0.0 & 0 & 0.0 & 0 & 0.0 \\
\hline \multirow{4}{*}{ Trip purpose } & Work & 0 & 0.0 & 4 & 9.1 & 2 & 3.6 & 1 & 3.4 \\
\hline & Study & 16 & 100.0 & 7 & 15.9 & 1 & 1.8 & 2 & 6.9 \\
\hline & Sport & 0 & 0.0 & 26 & 59.1 & 49 & 87.5 & 22 & 75.9 \\
\hline & Shopping & 0 & 0.0 & 7 & 15.9 & 4 & 7.1 & 4 & 13.8 \\
\hline
\end{tabular}

The sample of the red path is the one that mostly differs from the others. It is composed almost totally by boys aged to 20 and 30 . All are students who use the path for purposes related to the study. This result is not surprising due to the position of the path and the connection with the university campus. The samples of the other three paths are more varied 
in terms of gender, age and employment composition. More specifically, interviewed people along green path are prevalently males $(59.1 \%)$ than females. Half of them are aged between 31 and 50,35.1\% are aged between 21 and 30, and $13.6 \%$ between 51 and 60 . Most of them works as employee or freelancer, and almost 30\% are students. More than $30 \%$ of users declared to use the green path once a week, and $20.5 \%$ one every 10 days. The majority of users cycle along the green path for sport $(59.1 \%)$, followed by those who cycle for study $(15.9 \%)$ and shopping $(15.9 \%)$. In terms of trip purpose, the yellow and the blue paths are very similar. In fact, they are used prevalently for sport activities $(87.5 \%$ and $75.9 \%$ respectively). Roughly half of users uses both the yellow and the blue path once a week. Most of them are aged between 31 and 50, and slightly more than half are males.

\section{RESULTS}

In the following, in order to compare the cycle paths, some line charts highlighting the differences in users' perceptions were proposed. More specifically, the cycle paths have been compared in terms of: level of comfort on the whole path and in a specific point; degree of nuisance on the whole path and in a specific point; degree of protection in a specific point. In each chart, each line is related to a cycle path and it is colored according to the corresponding path.

\subsection{Comparison of cycle paths}

The comparison of users' perceptions about the level of comfort perceived on the whole part is reported in Fig. 3.

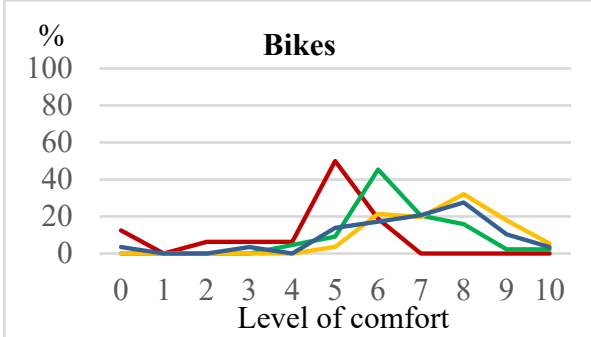

(a)

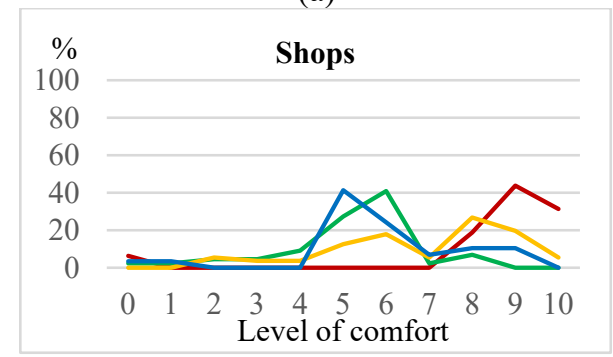

(c)

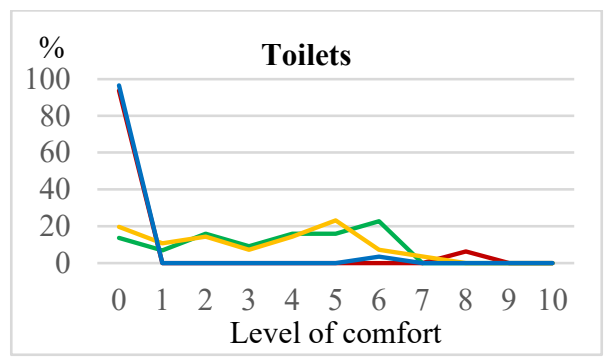

(b)

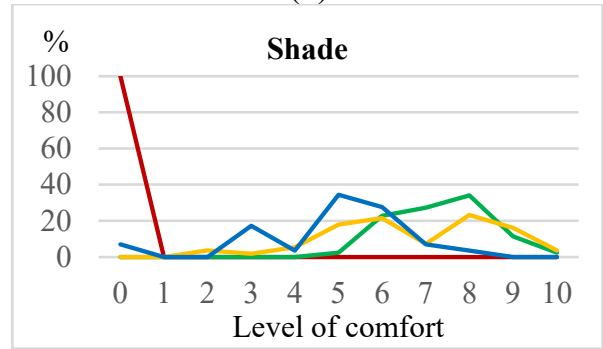

(d)

Figure 3: Level of comfort perceived on the whole path. (a) Other bikes; (b) Toilets; (c) Shops; and (d) Shade. (The evaluation scale ranges from 0 - "totally uncomfortable" to 10 - "totally comfortable".) 
In general, the presence of other bikes does not cause a particular discomfort in all the paths, even if for the red path about $50 \%$ of users expressed an intermediate level of comfort. On the contrary, low levels of comfort in relation with the toilets can be observed, and especially in red and blue path. The comfort level related to shady areas is acceptable for the yellow and the blue paths, very good for the green one, and very low for the red path. However, this last one is the path with the highest levels of comfort related to shops. In fact, it is the only one that do not cross green areas but has many commercial activities, even if also users of the other three paths did not express low level of comfort regarding this aspect. From the analysis of this first group of evaluated services, it emerges that the red path is perceived very differently from the others.

By observing the charts related to the degree of nuisance (Fig. 4), the most interesting results can be observed for the presence of pedestrians along the cycle paths. The highest degree of nuisance caused by the pedestrian flow is observed in the red path, followed by the green and yellow ones. This result is probably due to the characteristics of the paths and to the users' habits as well. In fact, the absence of the bikeway does not eliminate the pedestrians-cyclists' interactions on the red path. Similarly, the green path is shared. On the other hand, even if the bikeway of the yellow path is separated from the walkway, it is often improperly used by pedestrians.

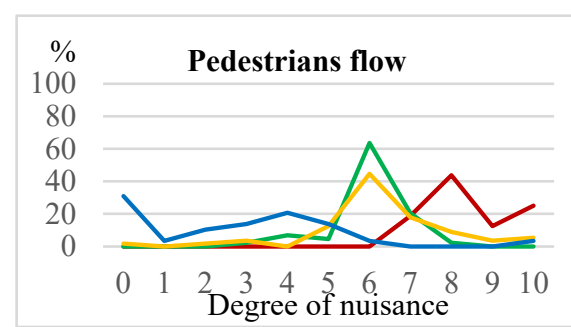

(a)

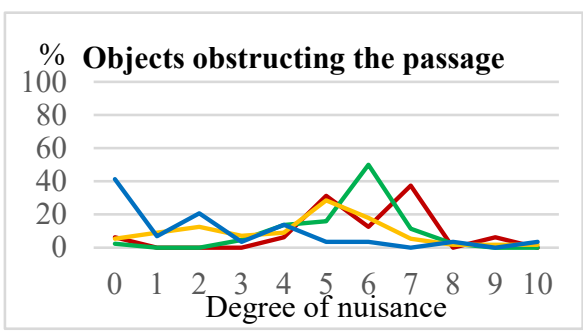

(c)

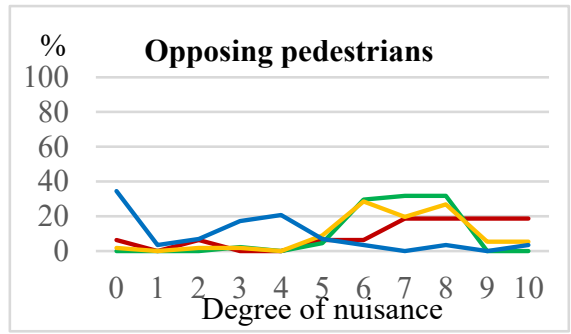

(b)

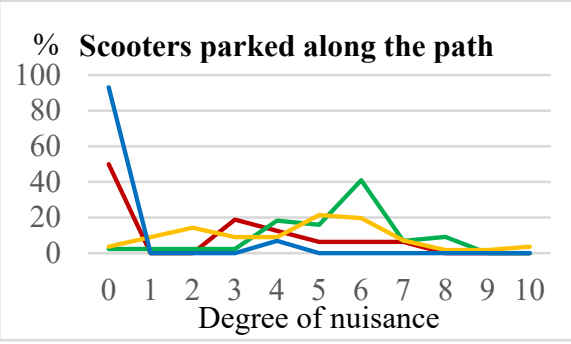

(d)

Figure 4: Degree of nuisance perceived on the whole path. (a) Pedestrian flow; (b) Opposing pedestrians; (c) Objects obstructing the passage; and (d) Scooters parked along the path. (The evaluation scale ranges from 0 - "absence of nuisance" to 10 - "high degree of nuisance".)

Regarding the degree of nuisance due to the objects obstructing the bikes' passage, no relevant differences result from the comparisons of the paths, even if the blue path was judged quite differently. In fact, while most of the users of all the paths expressed a degree of 
nuisance from 4 to 8 , the majority of the users of the blue path are not considerably disturbed by obstructing objects. Otherwise, the scooters parked along the path seem to be a problem for the blue and the red path.

Concerning the degree of nuisance in a specific point of the cycle path (Fig. 5), the users of the red path are those mostly annoyed for trucks and buses volume and traffic volume in general; this is an expected result, being this path an urban road registering relevant levels of congestion during the day. Anyway also the yellow and the green paths show considerable nuisance degrees regarding these aspects, while users of the blue path seem not suffering from traffic. Regarding pollution and noise nuisance, no relevant differences can be observed among the cycle paths, even if also in this case the blue path is the most virtuous one. Definitively, concerning this group of aspects, the blue path is the most performing alternative.

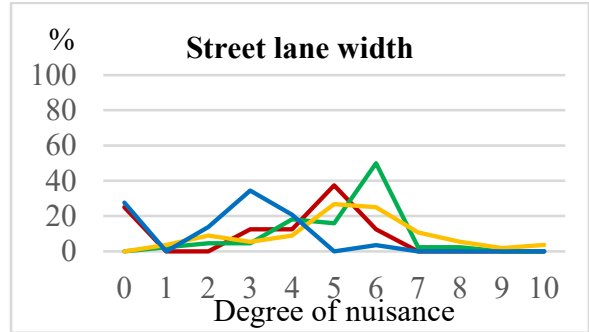

(a)

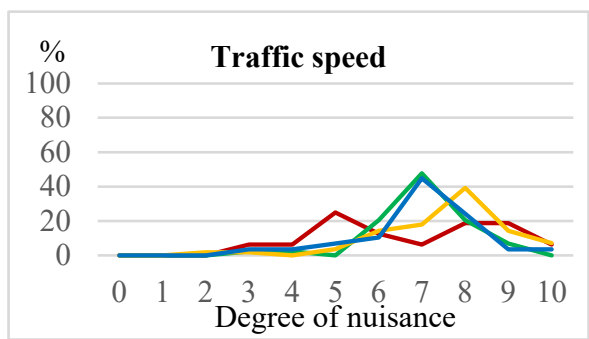

(c)

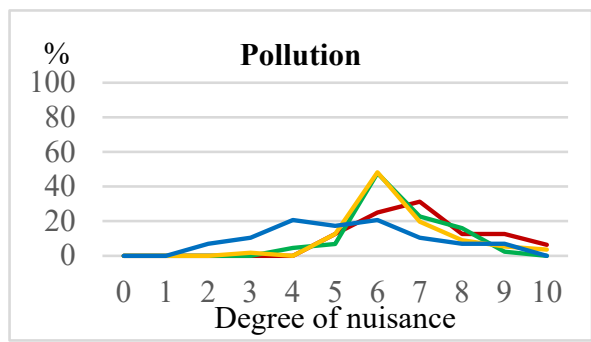

(e)

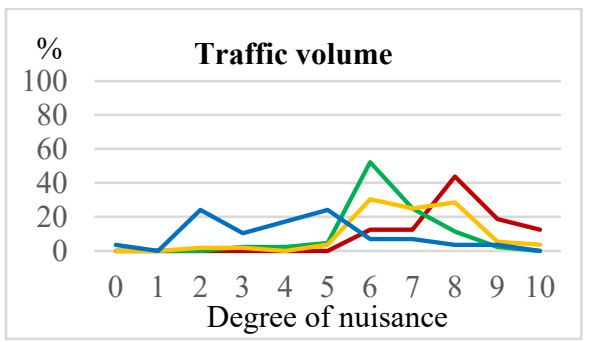

(b)

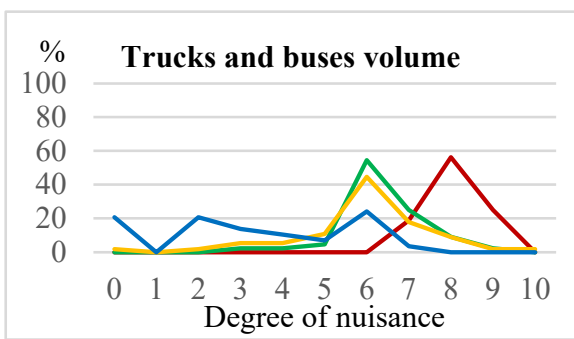

(d)

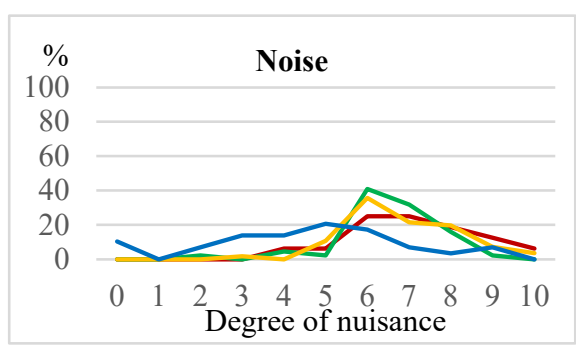

(f)

Figure 5: Degree of nuisance perceived on a specific point of the path. (a) Street lane width; (b) Traffic volume; (c) Traffic speed; (d) Trucks and buses volume; (e) Pollution; and (f) Noise. (The evaluation scale ranges from 0 - "absence of nuisance" to 10 - "high degree of nuisance".) 
In Fig. 6 the level comfort perceived on specific points of the cycle paths are shown. As expected the most critical issues occurred for the red path, and specifically concerning the perceived level of comfort with the temperature, lightning, odor and cleanliness. On the contrary, the other three paths seem to be little better concerning these aspects, and particularly the blue path for cleanliness and lighting in particular. Regarding the comfort related to environment closed to the path, perceptions of users is very similar among the four analyzed paths. Also landscape shows a quite similar tendency.

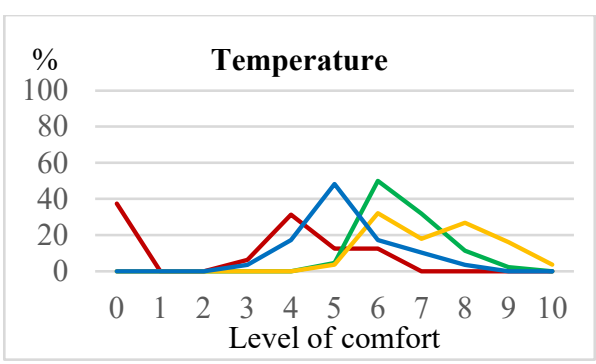

(a)

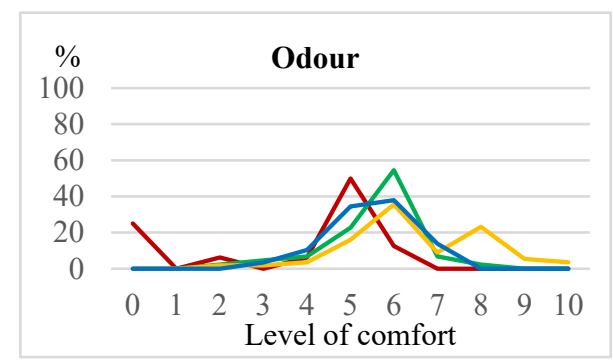

(c)

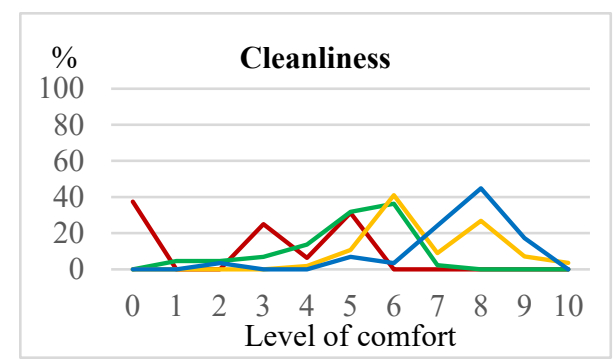

(e)

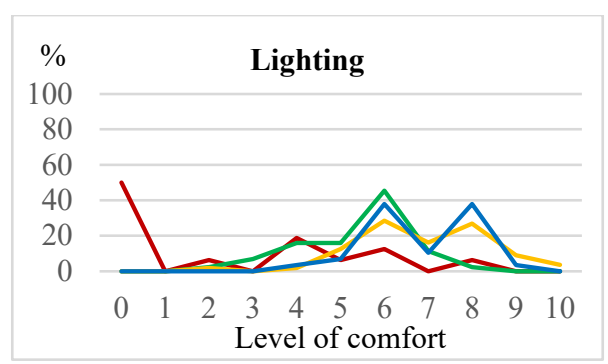

(b)

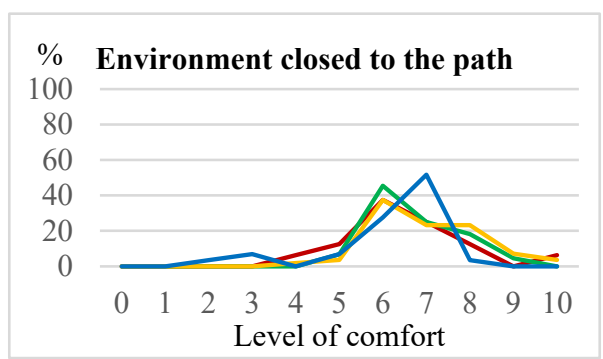

(d)

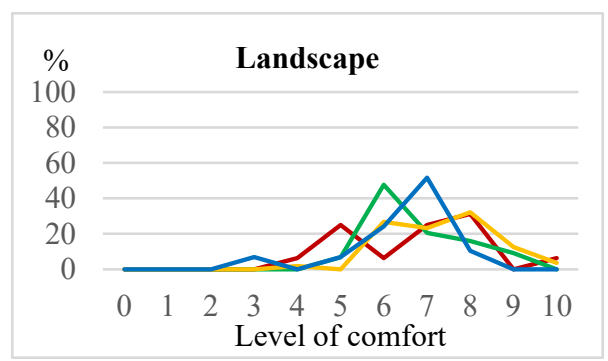

(f)

Figure 6: Level of comfort perceived on a specific point of the path. (a) Temperature; (b) Lighting; (c) Odor; (d) Environment closed to the path; (e) Cleanliness; and (f) Landscape. (The evaluation scale ranges from 0 - "totally uncomfortable" to 10 - "totally comfortable".)

The last group of charts (Fig. 7) allows to compare the cycle paths on the basis of users' perceptions of degree of protection on specific points of the paths. Most likely, the lack of a bikeway infrastructure with adequate equipment makes the red route much more vulnerable 
in terms of safety, as observable in Fig. 7(a)-7(c). However, the users of the other cycle paths do not express high degrees of protection in this sense, even if the blue path is the best in terms of protection from theft and robbery. Concerning protection from the weather, users of the yellow and the green paths show a certain perception of being protected, while all the users of the red path feel completely unprotected from the weather, as well as from the stray animals.

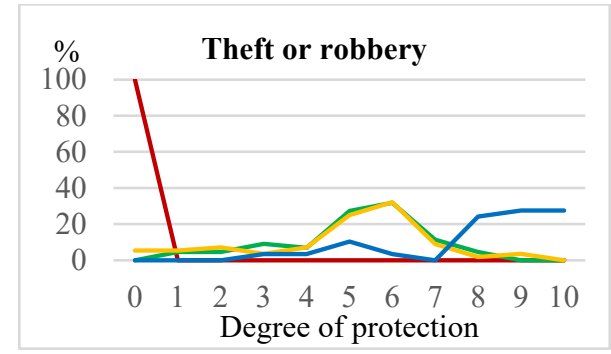

(a)

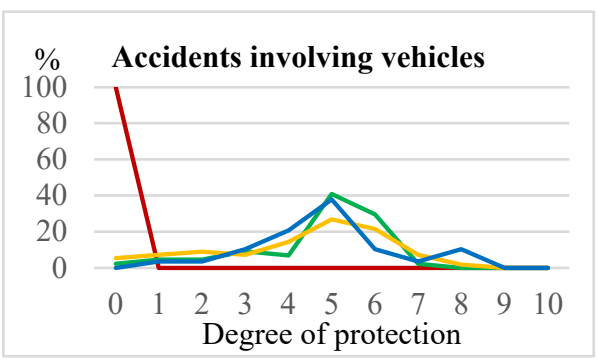

(c)

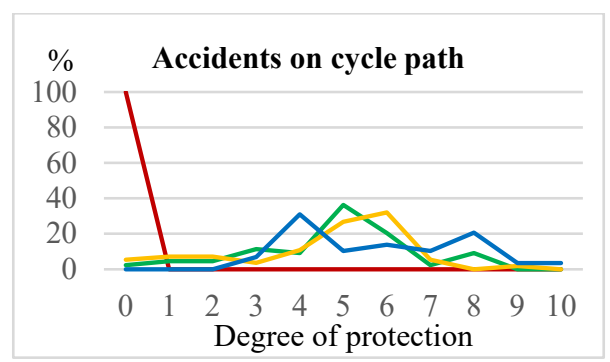

(b)

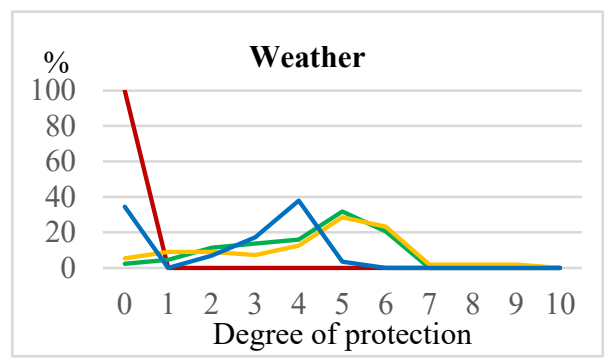

(d)

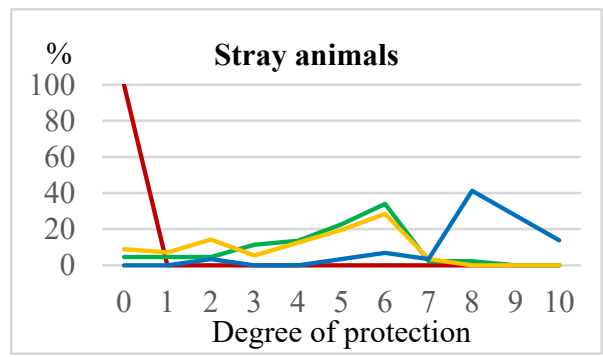

(e)

Figure 7: Degree of protection perceived on a specific point of the path. (a) Theft or robbery; (b) Accidents on cycle path; (c) Accidents involving vehicles; (d) Weather; and (e) Stray animals. (The evaluation scale ranges from $0-$ "absence of protection" to 10 - "high degree of protection".)

\section{CONCLUSIONS}

The aim of this paper was to propose an analysis of cyclists' perceptions of different paths in order to identify policy strategies for promoting cycling and inculcate the cycling culture as alternative to motorized transportation modes. Specifically, four different paths developing 
in an urban area of the southern Italy were considered as case study. A survey was conducted on samples of cyclists for collecting their comfort levels on some service aspects, their nuisance degrees relating to some aspects and protection degrees from some elements. The main difference between the four paths is that one path develops along an urban road, while the other paths are bikeways separated from the motorized-traffic; also other aspects such as the presence of green areas or shops differentiate the paths. In addition, the various paths are used by different types of cyclists. As suggested by Damant-Sirois et al. [9], it is fundamental to identify the different strategies to be applied according to the different types of cyclists. From the analysis of the cyclist' responses, interesting differences emerge between the various paths. As an example, the first path (the red) is the only path without a real cycling infrastructure, but it is largely used by students for walking or cycling, being the main route connecting the residential area with the university campus. As reported in Baglooe et al. [11], one way to promote the market share of the bicycle mode is to separate bicycles from motorized transport. However, enforcing bicycle lanes on congested roads, like the case of our red path, may degenerate the network as a whole. Maybe the best solution in this case would be an alternative cycling path connecting the residential area with the university campus. A future development of this work could be the proposal of such a bikeway, with adequate levels of comfort in terms of lighting and safety, as suggested by the users. An additional important strategy should be the reorganization of land uses in the university campus, giving priority to bikers and pedestrians instead to cars [8].

Finally, an aspect that unite the analyzed paths is surely the lack of connection with public transport system. The importance of integrating cycling with public transport or the encouragement of cycling is being emphasized by various countries in the world [5].

Definitively, we discovered that policies should be oriented towards the improvement of many different aspects, considering also the different types of cyclists. Surely the aspects concerning green or landscape are important and attractive for people cycling for recreational purposes. However, the cycling culture in urban areas has to be stimulated by focusing the attention on the aspects linked to the connection with public transport and other activities.

\section{ACKNOWLEDGEMENTS}

Support from "POR Calabria FESR-FSE 2014/2020 - Linea B Azione 10.5.12" is gratefully acknowledged. The authors would like to thank the students Gaetano Forgione, Marina Mingrone and Lucia Marte who collected the data for the elaboration of their degree thesis in Civil Engineering at the University of Calabria, Italy. The authors are very grateful to the Professor Alvaro Rodriguez-Valencia from the Universidad de los Andes, Bogotá-Colombia for his helpfulness and kindness to share the questionnaire.

\section{REFERENCES}

[1] Bellizzi, M.G., Eboli, L. \& Forciniti, C., Segregation vs interaction in the walkways: An analysis of pedestrians' perceptions. Research in Transportation Business and Management, 33, 100410, 2019. DOI: 10.1016/j.rtbm.2019.100410.

[2] Haustein, S., Koglin, T., Nielsen, T.A.S. \& Svensson, Å., A comparison of cycling cultures in Stockholm and Copenhagen. International Journal of Sustainable Transportation, 14(4), pp. 280-293, 2020. DOI: 10.1080/15568318.2018.1547463.

[3] Ma, L. \& Dill, J., Associations between the objective and perceived built environment and bicycling for transportation. Journal of Transport \& Health, 2(2), pp. 248-255, 2015. DOI: $10.1016 /$ j.jth.2015.03.002.

[4] TNS opinion \& social, special eurobarometer 422a - Quality of transport. European Union, 2014. 
[5] Saplığlu, M. \& Aydın, M.M., Choosing safe and suitable bicycle routes to integrate cycling and public transport systems. Journal of Transport \& Health, 10, pp. 236-252, 2018. DOI: $10.1016 /$ j.jth.2018.05.011.

[6] Rodriguez-Valencia, A., Rosas-Satizabal, D., Unda, R. \& Handy, S.L., The decision to start commuting by bike in Bogotá, Colombia: Motivations and influences. Travel Behaviour and Society, 24, pp. 57-67, 2021. DOI: 10.1016/j.tbs.2021.02.003.

[7] Doorley, R., Pakrashi, V., Szeto, W.Y. \& Ghosh, B., Designing cycle networks to maximize health, environmental, and travel time impacts: An optimization-based approach. International Journal of Sustainable Transportation, 14(5), pp. 361-374, 2020. DOI: $10.1080 / 15568318.2018 .1559899$.

[8] Fernández-Heredia, Á., Monzón, A. \& Jara-Díaz, S., Understanding cyclists' perceptions, keys for a successful bicycle promotion. Transportation Research Part A: Policy and Practice, 63, pp. 1-11, 2014. DOI: 10.1016/j.tra.2014.02.013.

[9] Damant-Sirois, G., Grimsrud, M. \& El-Geneidy, A.M., What's your type: A multidimensional cyclist typology. Transportation, 41(6), pp. 1153-1169, 2014. DOI: 10.1007/s11116-014-9523-8.

[10] Festa, D.C. \& Forciniti, C., Attitude towards bike use in Rende, a small town in South Italy. Sustainability, 11(9), 2703, 2019. DOI: 10.3390/su11092703.

[11] Bagloee, S.A., Sarvi, M. \& Wallace, M., Bicycle lane priority: Promoting bicycle as a green mode even in congested urban area. Transportation Research Part A, 87, pp. 102-121, 2016. DOI:/10.1016/j.tra.2016.03.004. 